\title{
Down-regulation of miR-133a-3p protects lung tissue against sepsis-induced acute respiratory distress syndrome by up-regulating SIRT1
}

Qin Hui ${ }^{1,2}$, Qi Zhang ${ }^{1}$, Xuan $\mathrm{Li}^{1}$, Kundi Wang ${ }^{1}$, Jing Zhang ${ }^{1}$, Zhongshu Zhou ${ }^{1,2}$

\author{
${ }^{1}$ Department of Pediatrics, China-Japan Friendship Hospital, Chaoyang District, \\ Beijing, China \\ ${ }^{2}$ Graduate School of Peking Union Medical College, Chinese Academy of Medical \\ Sciences, Beijing, China
}

Submitted: 17 September 2019

Accepted: 9 November 2019

Arch Med Sci

DOI: https://doi.org/10.5114/aoms.2020.94410

Copyright $\odot 2020$ Termedia \& Banach

\begin{abstract}
Introduction: MicroRNA-133a-3p (miR-133a-3p) is a potential gene regulator having an important role in the process of inflammation and lung injury. The present work studied the role of miR-133a-3p in sepsis-mediated acute respiratory distress syndrome (ARDS) and the mechanism involved.

Material and methods: $\mathrm{C} 57 \mathrm{BL} / 6$ mice were selected for the study. Protein expression of $\mathrm{Bcl}-2$, cleaved caspase- 3 and $\mathrm{Bax}$ was assessed by western blot analysis. Expression of mRNA was assessed by RT-PCR. Effects of inflammation were studied by myeloperoxidase (MPO) activity. Quantification of albumin was done by measuring the albumin conjugated with Evan's blue. The alveolar macrophages were separated from the lungs of mice by the bronchoalveolar lavage procedure and were submitted to sepsis challenge in vitro; the macrophages were treated with lipopolysaccharide (LPS).

Results: Treatment of LPS resulted in upregulation of miR-133a-3p in alveolar macrophages. Suppression of miR-133a-3p halted the over-expression of inflammatory cytokines in macrophages and caused remission of histopathologic changes. The ARDS lungs showed a decrease in levels of proinflammatory cytokines and an increase in levels of apoptotic protein, establishing the protective role for miR-133a-3p. The results suggested sirtuin 1 (SIRT1) as a potential target of miR-133a-3p in the macrophages, also showing that expression of SIRT1 was inversely associated with expression of miR-133a-3p. The protective effect of miR-133a-3p down-regulation in LPS-mediated alveolar macrophages and sepsis-induced ARDS could be corrected by a SIRT1 inhibitor.

Conclusions: Down-regulation of miR-133a-3p may exert a protective effect on lung tissue against sepsis-mediated ARDS by up-regulating the levels of SIRT1 via suppressing the inflammatory response and inhibiting the cellular apoptosis in lung tissues.
\end{abstract}

Key words: miR-133a-3p, sepsis injury, lungs, SIRT1.

\section{Introduction}

Sepsis is a serious problem having increasing incidence globally [1]. Sepsis is involved in infection and inflammation; it can lead to multiple organ failure and death $[2,3]$. The mortality rate remains high despite having a number of treatment approaches for sepsis [4]. Hence, there is an urgent need for developing new and effective treatment approaches for treating sepsis.

\author{
Corresponding author: \\ Dr. Zhongshu Zhou \\ Department \\ of Pediatrics \\ China-Japan \\ Friendship Hospital \\ Chaoyang District \\ 100029 Beijing, China \\ Phone/fax: \\ +86-13511037022 \\ E-mail: AnneBarajasoaf@ \\ yahoo.com
}


Acute respiratory distress syndrome (ARDS) is one of the dangerous forms of acute lung injury characterized by an inflammatory response of the respiratory system. ARDS leads to respiratory failure along with damage to the parenchyma of lungs $[5,6]$. Both ARDS and acute lung injury are associated with sepsis, making it as a major risk factor [7]. Lungs are the most vulnerable to sepsis and hence contribute primarily to deaths in cases of respiratory failure [8]. It is reported that patients diagnosed with sepsis-mediated ARDS have high severity for illness which also increases the rate of mortality [5]. The mechanisms involved in ARDS and ALI include higher infiltration rates of proteins, leucocytes, inflammation and elevated oxidative stress [9]. Therapies improving pathologic conditions of ARDS would be beneficial [10].

Sirtuins (SIRT) have been reported to show diverse roles in various physiological processes including heart disease, neurodegenerative disorders, cancer and inflammation [11]. There are 7 members of the SIRT family (SIRT1-SIRT-7); these SIRT members vary in structure, functions and localization. Among all the members SIRT1 is the most widely studied $[12,13]$. SIRT1 is found to regulate the expression of inflammatory mediators in lungs of cigarette smokers. SIRT1 regulates the expression of nuclear factor $\kappa \mathrm{B}(\mathrm{NF}-\mathrm{\kappa B})$ and p65 in both in vitro and in vivo models; it regulates acetylation of NF-KB, which is crucial for the release of proinflammatory mediators [14].

MicroRNAs are small non-coding RNAs, responsible for suppression of the transcription and translation process via binding directly to the target site and can regulate gene expression [15]. MicroRNAs play a potential role in regulating the cellular process, differentiation, metabolism, proliferation and apoptosis $[16,17]$. MiRNAs have also been identified to play a potential role in pulmonary fibrosis, lung cancer, tuberculosis, and bronchial hypersensitivity. MiR-133a-3p belongs to the miR-133 family of miRs. MiR-133a-3p occurs in the human genome with different copies such as miR-133a-1, -2 and is present on the $18^{\text {th }}$ and $20^{\text {th }}$ chromosome [18]. To date multiple functions of miR-133a-3p have been established such as regulation of myoblasts [19], inhibition of proliferation of cardiomyocytes [20], in malignancies such as lung cancer [21], colon cancer [22] and bladder cancer [23]. Recently miR-133a-3p along with $155-5 p$ has been profiled in diagnosis and prognosis of sepsis [24].

In a study recently involving differentiation of cardiomyocytes in developing heart, it was evidenced that the 3'UTR region of SIRT1 had a potential binding site for miR-133a, and the binding of miR-133a regulated the expression of SIRT1. The report ultimately confirmed that expression of SIRT1 is regulated by miR-133a [25]. However, the role of miR-133a-3p in sepsis-mediated ARDS has not been studied. The present study evaluated the role of miR-133a in sepsis-induced ARDS and also confirmed the pathway involved.

\section{Material and methods}

\section{Animals and ethics statement}

For the study adult male C57BL/6 mice aged between 6 and 8 weeks were used and procured from Jackson Laboratory USA. All the experimental protocols were in agreement with ethical guidelines from the Ministry of Health, China. The protocols were approved by the animal ethical committee of China-Japan Friendship Hospital, Beijing, China. The animals were housed under standard room temperature conditions $\left(24-25^{\circ} \mathrm{C}\right)$ with a 12-hour light and dark cycle. The animals were fed with water and pallet diet ad libitum.

\section{Histological examination}

At the end of the study the mice were sacrificed and the lung specimens were isolated. The lung tissues were fixed in paraffin and sections of $5 \mu \mathrm{m}$ were obtained using a rotary microtome. The sections of lung tissues were stained with hematoxylin and eosin.

\section{Western blotting analysis}

Part of lung tissues were used for histological studies and the remains were used further for studying the expression of proteins. The lung tissues were homogenized in a tissue homogenizer using PBS as vehicle and then centrifuged; the supernatants were used for expression of proteins. To evaluate the protein levels of Bcl-2, cleaved caspase- 3 and Bax, western blot analysis was done. About $50 \mu \mathrm{g}$ of protein was submitted to SDS-PAGE followed by transfer to PVDF membranes. The membranes were further incubated at $4^{\circ} \mathrm{C}$ for $12 \mathrm{~h}$ along with $\mathrm{I}^{\mathrm{ry}}$ antibodies specific to Bcl-2 (1: 1000, Abcam, USA), Bax (1: 1000, Abcam, USA), cleaved caspase-3 (1:1000, Cell Signaling Tech., USA), $\beta$-actin (1: 1000, Cell Signaling Tech., USA), SIRT1 (1 : 1000, Abcam, USA). The membranes were incubated with horseradish peroxidase secondary antibodies $(1: 3000)$ for $60 \mathrm{~min}$. The bands of protein obtained were viewed using the ECL Western Blotting Substrate kit (Abcam USA). Densitometric analysis was done for expression of target proteins against the loading control.

\section{RT-PCR analysis}

For RT-PCR study, 500 ng of RNA was extracted from the isolated lung tissues using an RNA isolation kit (Sigma Aldrich USA). The isolated RNA was subjected to reverse transcription using the 
Table I. Primer sequences used for the study

\begin{tabular}{|c|c|c|}
\hline Name of protein & Forward & Reverse \\
\hline GAPDH & 5'-GTGTTCCTACCCCCAATGTG-3' & 5'-CATCGAAGGTGGAAGAGTGG-3' \\
\hline IL-1 $\beta$ & 5'-GGGCCTCAAAGGAAAGAATC-3' & 5'-TACCAGTTGGGGAACTCTGC-3' \\
\hline TNF- $\alpha$ & 5'-CGTCAGCCGATTTGCTATCT-3' & 5'-CTTGGGCAGATTGACCTCAG-3' \\
\hline IL-6 & 5'-GGGACTGATGCTGGTGACAA-3' & 5'-TCCACGATTTCCCAGAGAACA-3' \\
\hline miR-133a-3p & 5'-CGGGGTACCCAGTTGGGTGTTGAAGGAGA-3' & 5'-CCGCTCGAGAGCACTACACGGCCAATG-3' \\
\hline U6 & 5'-GTGCTCGCTT & CAGCACATAT-3' \\
\hline
\end{tabular}

TonkBio RT reagent Kit (TonkBio, USA). The cDNAs were processed and amplified using Teloprime full length cDNA amplification kit V2 (Lexogen Austria). The study was done using a real-time system, Bio-Rad USA. The PCR conditions were as follows: denaturation of proteins for $30 \mathrm{~s}$ at $95^{\circ} \mathrm{C}$, normalization for $10 \mathrm{~s}$ at $60^{\circ} \mathrm{C}$, followed by elongation for $20 \mathrm{~s}$ at $72^{\circ} \mathrm{C}$; about 30 cycles were done for the whole processing. The expression of mRNA was normalized against miRNA levels of U6 and mRNA levels of GAPDH. The sequences of primers used are described in Table I.

\section{Myeloperoxidase activity}

For studying the effects of inflammation in lungs, MPO activity was assessed. The animals were sacrificed and the lung tissues were harvested; the lungs were weighed and stored at $-80^{\circ} \mathrm{C}$. The lung tissues were analyzed for myeloperoxidase (MPO) using an MPO activity assay kit (Abcam, USA) following the supplied instructions.

\section{Infiltration of Evan's blue dye}

The quantification of albumin was done by measuring the albumin conjugated with Evan's blue (Sigma Aldrich USA). Infiltration of Evan's blue dye $(20 \mathrm{mg} / \mathrm{kg})$ was done by injecting it via the retro-orbital plexus of mice. After injecting, the mice were rested for $60 \mathrm{~min}$ and were then sacrificed. The lungs were subjected to perfusion using phosphate buffer saline through the right ventricle, removed and weighed and were then homogenized in a tissue homogenizer in $500 \mu \mathrm{l}$ of formaldehyde. The homogenates were incubated at $37^{\circ} \mathrm{C}$ for $24 \mathrm{~h}$ followed by centrifugation at $10000 \mathrm{rpm}$ for $20 \mathrm{~min}$. The supernatants were collected and absorbance was recorded at $620 \mathrm{~nm}$. The quantification of Evans blue dye in lungs was done by creating a standard calibration curve.

\section{Isolation of alveolar macrophages from lungs}

The alveolar macrophages were separated from the lungs of mice by a bronchoalveolar lavage procedure, counted and were suspended again in DMEM/F-12 medium (Thermo Fisher, USA) pre-supplemented with fetal bovine serum (10\%), penicillin $(100 \mathrm{U} / \mathrm{ml})$ and streptomycin $(100 \mathrm{U} /$ $\mathrm{ml}$ ) (Sigma Aldrich USA). The isolated alveolar macrophages were submitted to sepsis challenge in vitro, in which they were treated with lipopolysaccharide (LPS) $(1 \mu \mathrm{g} / \mathrm{ml})$ (Sigma Aldrich USA) for $4 \mathrm{~h}$. Before inducing the macrophages, the medium was added with SIRT1 and EX527 (1 $\mu \mathrm{M}) 24 \mathrm{~h}$ prior to LPS stimulation.

\section{Development of burn sepsis model in mice}

For the in vivo study, C57BL/6 mice aged 6 to 8 weeks were used and were anesthetized with injection of sodium pentobarbital $(50 \mathrm{mg} / \mathrm{kg}$ ); the back area of mice were shaved to remove the hairs. The shaved back area of $2 \mathrm{~cm}^{2}$ was exposed to steam for $8 \mathrm{~s}$ for burning the skin for sepsis. The burned back region was cooled at room temperature for $5 \mathrm{~min}$; the surface area was incubated with a suspension of Pseudomonas aeruginosa bacteria $(50 \mu \mathrm{l})$ using a microinjector. Immediately after the burning, the mice were injected with normal saline via the intraperitoneal route $(1 \mathrm{ml}$ $20 \mathrm{~g})$. In the process of establishing the burn sepsis model, miR-133a-3p antagomir (80 mg/kg/ day) was injected into the caudal vein for 3 continuous days. For inhibition, the mice were injected with normal saline, SIRT1 or EX527 (5 mg/kg).

\section{Enzyme-linked immunosorbent assay}

After $24 \mathrm{~h}$ of LPS stimulation, the mice were subjected to anesthesia using pentobarbital injection (30 mg/kg). Blood was collected and serum was extracted followed by analysis of tumor necrosis factor- $\alpha$ (TNF- $\alpha$ ) using an ELISA kit (Thermo Fisher USA).

\section{Transfection of macrophages}

For the purpose of transfecting the cells, miR133a-3p agomir or miR-133a-3p antagomir and their respective control RNAs were used (Qiagen). The macrophagic cells received transfection of 
scrambled negative control miR-133a-3p, miR$133 a-3 p$ antagomir or agomir using a transfecting reagent (X-tremegene, Sigma Aldrich USA). Briefly, about $20 \mu \mathrm{M}$ of miR-133a-3p antagomir or agomir was added and mixed with $12 \mu \mathrm{l}$ of transfection reagent in about $100 \mu \mathrm{l}$ of culture medium for $10 \mathrm{~min}$ at room temperature conditions to obtain the transfection complex. The macrophages were incubated along with complexes for $48 \mathrm{~h}$.

\section{Luciferase assay}

The 3'UTR region fragment of SIRT1 possessing the binding site for miRNA was amplified with the help of PCR; the CDNA template obtained from the macrophage RNA was used for the same. The mutant 3'UTR and the wild type 3'UTR having the potential binding sites for the sequence of miR$133 a-3 p$ were cloned downstream of the firefly luciferase gene in the pGL3 vector. The cells received co-transfection of miR-133a-3p or control miRNA. After $48 \mathrm{~h}$ from transfection, the cells were washed in phosphate buffer saline and the luciferase activity was measured with the help of the dual luciferase reporter assay system.

\section{Statistical analysis}

The statistical analysis was performed using GraphPad prism software. All the results are pre-

A

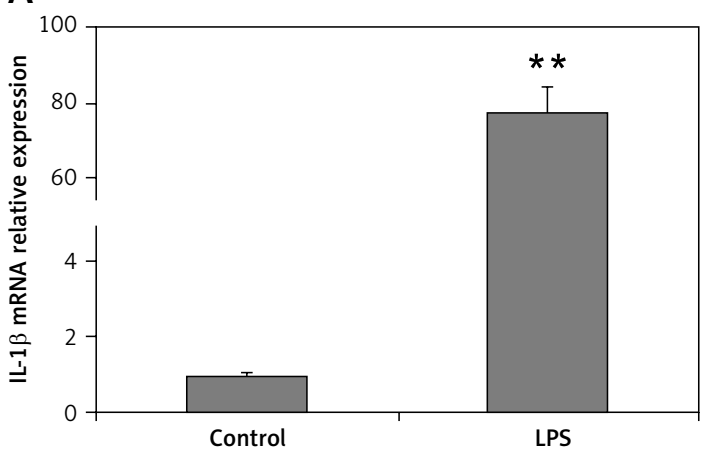

C

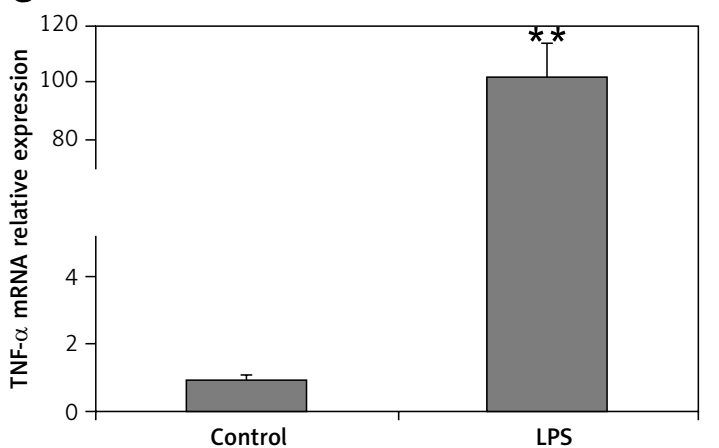

sented as mean $\pm \%$ RSD $(n=3)$. The statistical differences were established by performing the Mann-Whitney $U$-test. $P$-value $<0.05$ was considered as significant.

\section{Results}

\section{LPS stimulation increases the level of miR-133a-3p in macrophages}

LPS is identified to be one of the main factors in induction of acute lung injury mediated ARDS with alveolar macrophages being the main target cells. The results of RT-PCR suggested that the mRNA levels of TNF- $\alpha$, interleukin 6 (IL-6) and IL-1 $\beta$ were upregulated significantly in macrophages when stimulated with LPS $(100 \mathrm{ng} / \mathrm{ml})$ compared to the control (Figures $1 \mathrm{~A}-\mathrm{C}$ ). The miR133a-3p levels were also upregulated significantly after LPS treatment (Figure $1 \mathrm{D}$ ). The findings of the experiment suggested a potential role of miR$133 a-3 p$ in LPS-mediated upregulation of inflammatory cytokines in macrophages.

\section{miR-133a-3p is essential for LPS-mediated upregulation of inflammatory cytokines in macrophages}

To evaluate the role of miR-133a-3p in LPS-mediated upregulation of inflammatory cytokines,

B

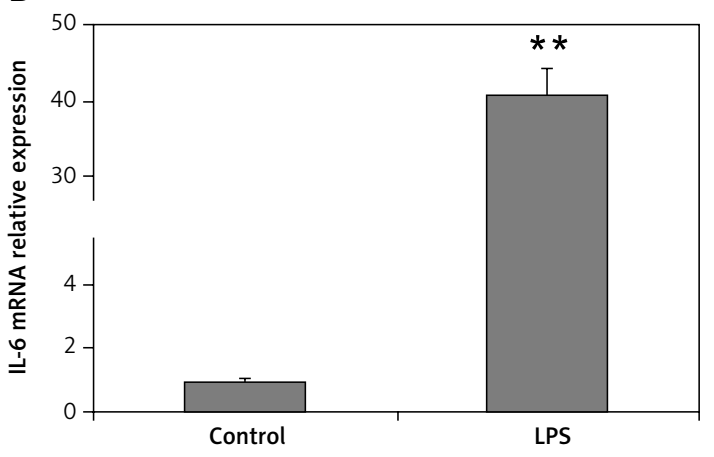

D

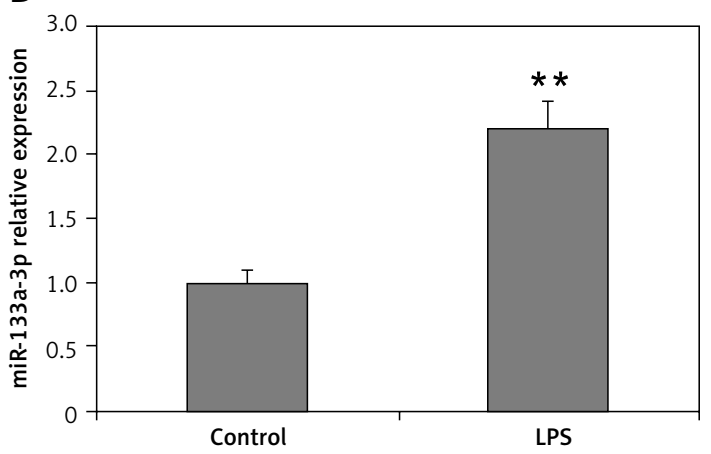

Figure 1. Levels of proinflammatory cytokines and miR-133a-3p in alveolar macrophages after stimulation with LPS. A-C - RT-PCR evaluation showing relative mRNA levels of IL-1 $\beta$, IL- 6 and TNF- $\alpha$ in alveolar macrophages after stimulation with LPS. D - miR-133a-3p levels in alveolar macrophages after stimulation with LPS

The data presented show means $\pm S E(n=6) ;{ }^{* *} p=0.01$ versus control. 
the alveolar macrophages were transfected with miR-133a-3p antagomir or agomir, so as to increase or decrease the expression levels of miR$133 a-3 p$. As presented in Figure $2 \mathrm{~A}$, the mRNA levels of IL-1 $\beta$ in macrophages increased significantly after treatment of LPS. The outcomes also suggested that the pre-exposure of miR-133a-3p antagomir decreased the mRNA levels significantly. It was also found that the mRNA levels of IL- 6 and TNF- $\alpha$ decreased significantly with a decrease in levels of miR-133a-3p (Figures 2 $B, C)$. Furthermore, it was evidenced that, when the levels of miR-133a-3p were upregulated, it resulted in increased mRNA levels of TNF- $\alpha$, IL- 6 and IL-1 $\beta$, as shown in Figures $2 \mathrm{D}$ and F. Altogether, the results of the experiment confirmed that miR-133a-3p is crucial for LPS-mediated upregulation of inflammatory cytokines in macrophages.

\section{Downregulated miR-133a-3p plays}

a protective role in mouse ARDS

The experiments so far have confirmed that miR-133a-3p is essential in upregulation of LPS-mediated pro-inflammatory cytokine. In the study expression of miR-133a-3p in the mouse model of sepsis was suppressed to study the role of miR-133a-3p in lung injury. The results (Figure $3 \mathrm{~A}$ ) demonstrated that treatment of miR133a-3p antagomir in C57BL/6 mice significantly decreased lung congestion, edema and hemorrhage compared to the negative control and antagomir group. The results of ELISA suggested that the expression of TNF- $\alpha$, which is a pro-inflammatory factor, increased significantly in lung tissues of sepsis-induced mice compared to the sham group, and the miR-133a-3p antagomir reversed the elevated levels of TNF- $\alpha$ (Figure $3 \mathrm{~B}$ ). The MPO activity was measured for assessing the activity of neutrophils in the lungs. The results are shown in Figure $3 \mathrm{C}$; the treatment of miR-133a-3p antagomir decreased the elevated MPO activity significantly in lungs of mouse with sepsis. The infiltration study of Evan's blue dye suggested that the treatment of miR-133a$3 p$ antagomir decreased the protein leakage in lungs of sepsis-induced mice (Figure 3 D). The protein levels of Bax and cleaved caspase- 3 were elevated significantly in the lung tissues of sepsis-induced mice; the treatment of miR-133a$3 p$ antagomir decreased the levels of protein significantly (Figure $3 \mathrm{E}$ ). The experiments also confirmed decreased levels of $\mathrm{Bcl}-2$ protein in sepsis-induced lung tissues; the treatment of miR-133a-3p antagomir increased the levels of $\mathrm{BCl}-2$ in lungs of sepsis-induced mice (Figure $3 \mathrm{E}$ ). These findings suggest an attenuating role of miR-133a-3p in septic mice.

\section{SIRT1 is a direct target of miR-133a-3p in macrophages}

MicroRNAs have been found to function mainly by regulating the target genes. With the possibility to identify the target genes of miR-133a-3p in alveolar macrophages, gene databases such as miRanda, TargetScan and PicTar were explored. The databases suggested that the SIRT1 gene had a favorable target site for miR-133a-3p in the $3^{\prime}$-UTR region. The database showed a match between the target site and the seed region of miR-133a-3p (Figure 4 A). As demonstrated, the luciferase activity was decreased significantly by miR-133a-3p mimics in the $3^{\prime}$-UTR of wild type, but the activity remained unsuppressed in the presence of the reporter transfected with mutant $3^{\prime}$-UTR, suggesting direct binding of miR-133a-3p to the $3^{\prime}$-UTR region of SIRT1 (Figure $4 \mathrm{~B}$ ). Also it was evidenced that upregulation of miR-133a$3 p$ in macrophages suppressed the expression of SIRT1 at protein as well as at mRNA levels, whereas the inhibition of miR-133a-3p elevated the expression of SIRT1 (Figures 4 C, D). These findings suggested SIRT1 as a favorable target of miR133a-3p which inhibits its levels.

\section{Inhibition of SIRT1 halts the protective effect of miR-133a-3p antagomir}

Outcomes of the study until now have confirmed that SIRT1 is a favorable target of miR133a-3p. To confirm this, a SIRT1 inhibitor (EX527) was used to suppress the activity of SIRT1 and its role in upregulation of inflammatory cytokine in LPS-mediated alveolar macrophages. The results showed that (Figure $5 \mathrm{~A}$ ) the decreased mRNA levels of IL-1 $\beta$ in macrophages after LPS treatment were attenuated significantly by the SIRT1 inhibitor EX527 (Figure 5 A), which also corrected the decreased mRNA levels of TNF- $\alpha$ and IL- 6 (Figures $5 \mathrm{~B}, \mathrm{C})$. These findings confirm that suppression of SIRT1 is essential in LPS-induced lung injury in alveolar macrophages through miR-133a-3p and also SIRT1 could play an important protective role.

\section{The attenuating effect of miR-133a-3p antagomir in sepsis-induced lung is SIRT1 dependent}

The findings have confirmed that decreased miR-133a-3p levels play an attenuating role in septic lungs in mice. The study further evaluated whether the protective role of SIRT1 is via upregulation of SIRT1 levels or not. For this, a SIRT1 inhibitor in sepsis-induced ARDS mice was used. It was found that treatment with miR-133a-3p antagomir + EX527 in mice aggravated the edema and lung congestion more significantly compared to the miR-133a-3p antagomir group (Figure $6 \mathrm{~A}$ ). 
A

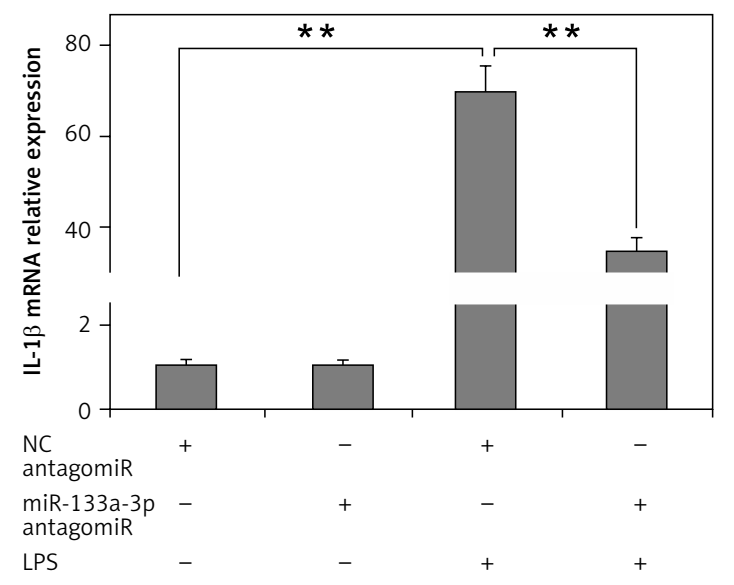

C

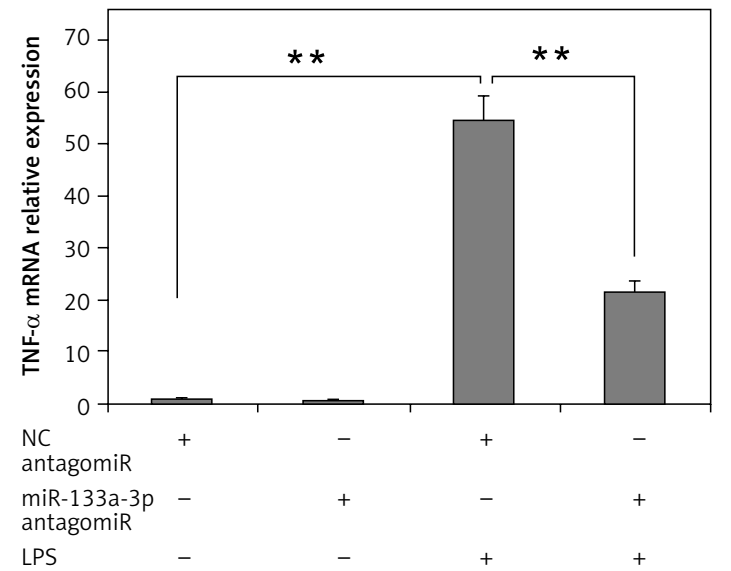

E

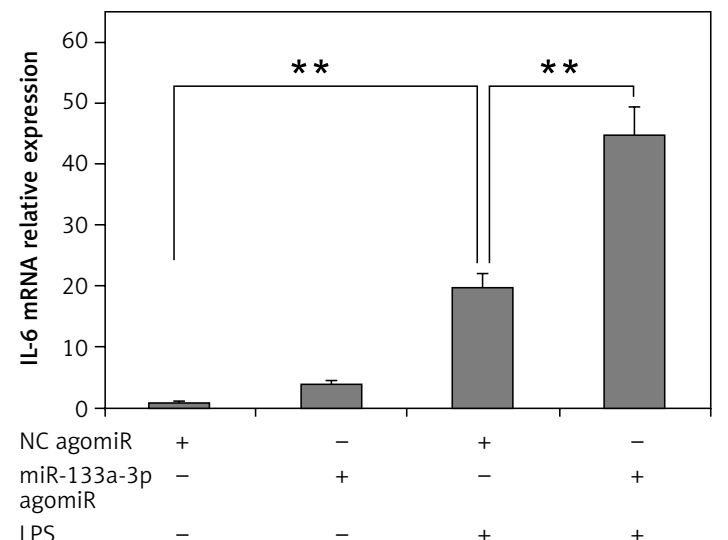

B



D

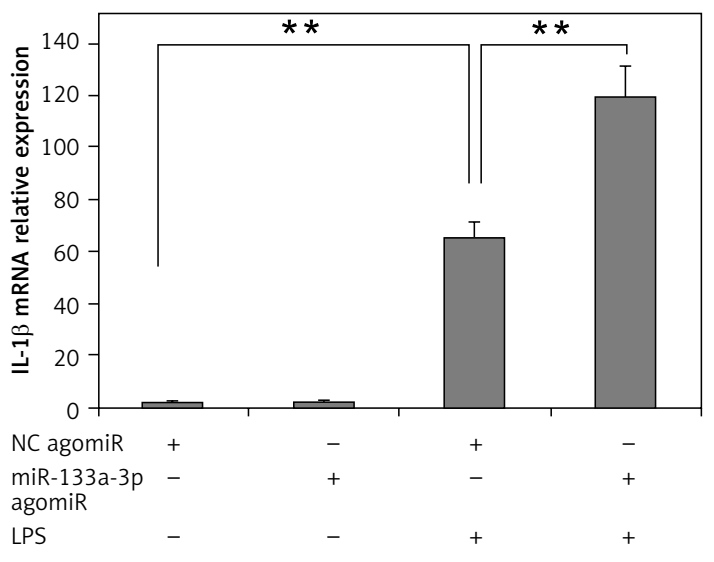

$\mathrm{F}$



Figure 2. Results of miR-133a-3p treatment on LPS-mediated over-expression of inflammatory cytokines in alveolar macrophages. A-C - RT-PCR evaluation demonstrating the mRNA expression levels of IL-1 $\beta$, TNF- $\alpha$ and IL-6 in alveolar macrophages in presence and absence of miR-133a-3p antagomir after LPS stimulation. D-F - Results of RT-PCR demonstrating the mRNA levels of IL-1 $\beta$, TNF- $\alpha$ and IL- 6 in alveolar macrophages in presence and absence of miR-133a-3p agomir after LPS stimulation

The data presented show means $\pm S E(n=6) ;{ }^{* *} p=0.01$ versus control. (NC) negative control. 
A

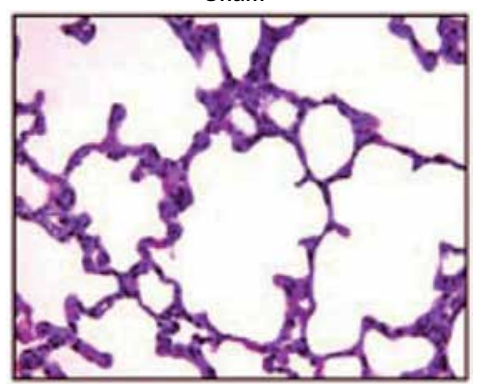

B

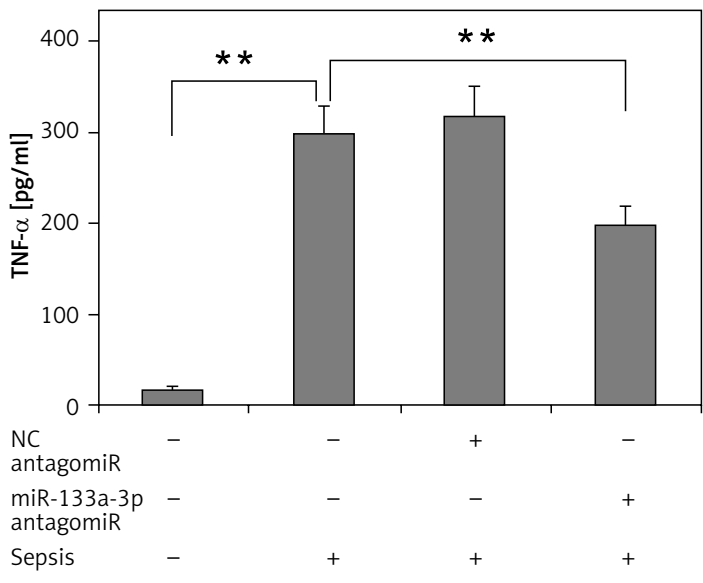

D

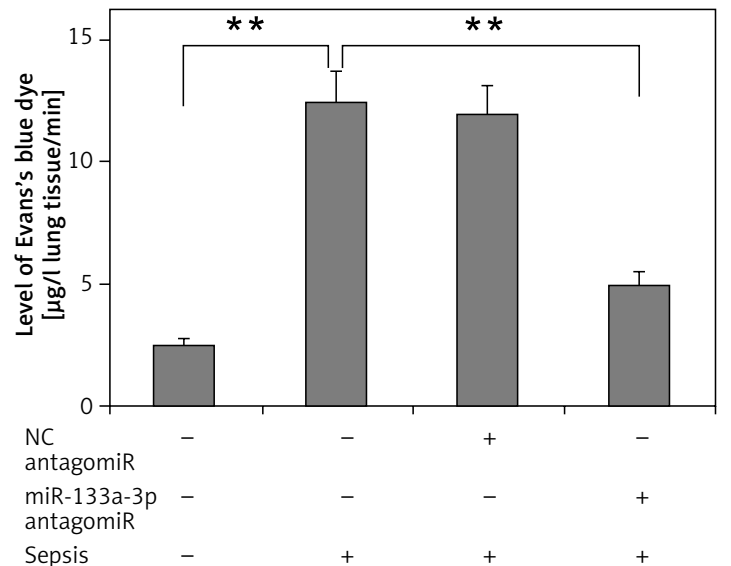

NC antagomiR

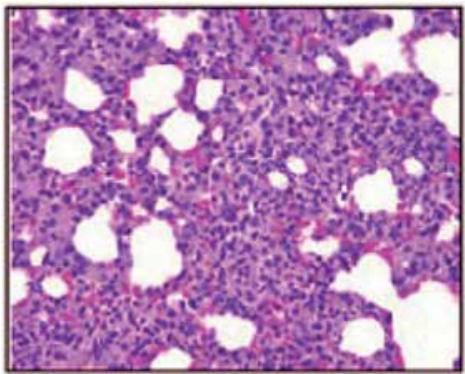

C



$E$

Cleaved caspase

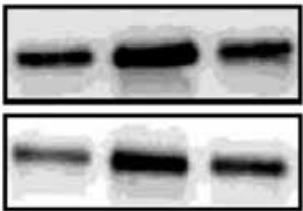

$\mathrm{Bcl}-2$

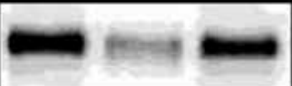

Actin

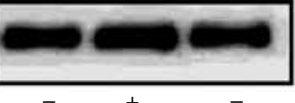

NC antagomiR

miR-133a-3p antagomiR

Sepsis

Figure 3. Results of miR133a-3p in acute sepsis-induced acute respiratory distress syndrome. A - Results of hematoxylin and eosin (H\&E) staining depicting the morphological changes in sepsis-induced lung tissue after $24 \mathrm{~h}$ of injury. The negative control miR-133a-3p antagomir exposed sepsis injury group (NC antagomir) exhibited increase in lung edema, hemorrhage in alveoli, infiltration of neutrophils and damaged epithelial/endothelial cell structure compared to the sham operated group. miR-133a-3p antagomir exposed sepsis animals (miR-133a-3p antagomir) exhibited significant attenuation in morphology of lung tissues. Scale bar, $50 \mu \mathrm{m}$. B - ELISA study exhibiting the effect of miR-133a-3p antagomir on levels of TNF- $\alpha$ in sepsis-induced lung injury after $24 \mathrm{~h}$ of treatment. C - Activity of MPO in sepsis-induced lung injury $24 \mathrm{~h}$ after miR-133a-3p antagomir treatment in every treatment group was recorded. D - Levels of Evan's blue dye evaluated in each group and treatment of miR-133a-3p antagomir caused a significant reversal and elevated the levels of Evan's blue dye mediated by sepsis injury after $24 \mathrm{~h}$ of treatment. $\mathrm{E}$-Western blot analysis exhibiting the effect of miR-133a-3p on expression levels of protein, i.e. cleaved caspase-3, $\mathrm{Bcl}-2$ and Bax in sepsis injury lung tissues after $24 \mathrm{~h}$ of treatment

Data are presented as means \pm SE $(n=6) ;{ }^{* *} p<0.01$ compared to sham operated group. 


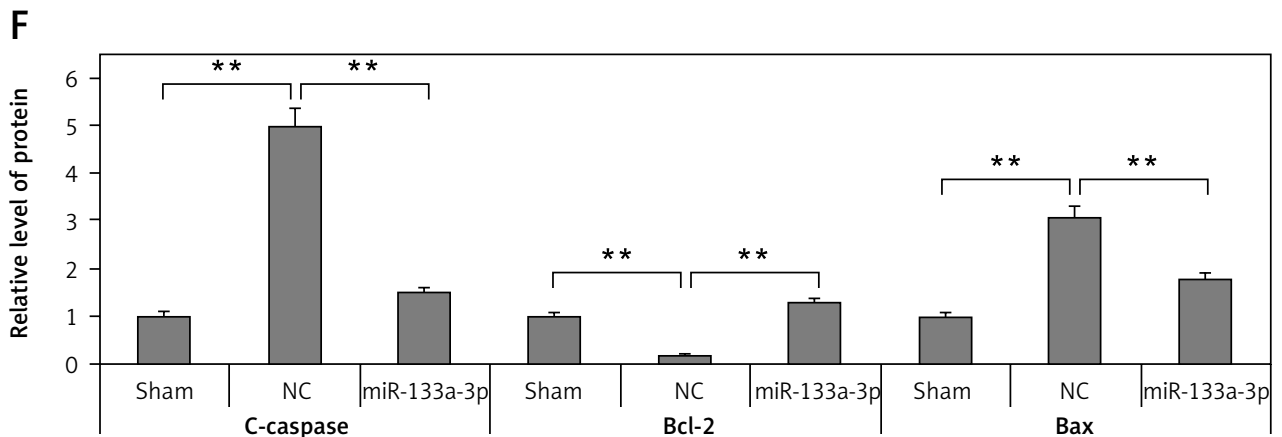

Figure 3. Cont. $\mathrm{F}-$ Quantitative analysis results for relative expression of proteins $\mathrm{C}$-caspase, $\mathrm{BCl}-2$ and $\mathrm{Bax}$ Data are presented as means $\pm S E(n=6)$; ${ }^{* *} p<0.01$ compared to sham operated group.

The levels of TNF- $\alpha$ were elevated significantly after receiving treatment with EX527 (Figure 6 B). The results of MPO activity and protein leakage increased significantly after receiving the EX527 treatment (Figures 6 C, D). The EX527 treatment reduced the levels of Bax of caspase- 3 and increased $\mathrm{Bcl}-2$ after the macrophages were treated with miR-133a-3p antagomir (Figure $6 \mathrm{E}$ ). The results hence suggest that miR-133a-3p exerts an attenuating role in the mouse sepsis model via upregulating the level of SIRT1.

\section{Discussion}

Sepsis is one of the most common factors in ALI/ARDS. It occurs due to a favorable interaction between invading microbes and the immune system of the host, producing an acute inflammatory as well as coagulation reaction [4, 26, 27]. Sepsis is accompanied by a serious systemic inflammatory response, which leads to damage of vital organs [28]. ARDS is a state showing acute respiratory failure and is characterized by diffusive infiltration and hypoxemia [29]. Studies have reported that the rate of sepsis-mediated mortality in subjects with ALI/ARDS has declined in the last 10 years but still the rate remains near to $40 \%$, which is still critical $[30,31]$. In sepsis-mediated lung injury, a number of inflammatory cytokines are liberated in the extracellular and intracellular matrix which leads to damage of alveolar membrane and dysfunctioning of alveolar capillary diffusion, leading to hypoxemia [19, 32]. Previous studies have demonstrated that migration and activation of the monocyte macrophage system cause over-release of inflammatory mediators such as TNF- $\alpha$, IL- 6 and IL- $1 \beta$, which are reported to be significant inducers of tissue injury and dysfunction [33-35].

Growing studies have shown that some specific genes play a vital role in the prognosis and pathogenesis of ARDS and sepsis. Interestingly, miRs have attracted major attention of researchers as being possible therapeutic targets for halting the progression of ARDS [16, 36]. miR-133a-
$3 p$ belongs to the miR-133 family of miRs; it is a multifunctional mRNA expressed in a number of pathologic conditions. The miR-133 family of miRs has been found to regulate the proinflammatory factors [37]. Production of proinflammatory cytokines is important for sepsis-mediated ARDS. It is found that both TNF- $\alpha$ and IL- $1 \beta$ are upregulated in patients reported for sepsis. Targeting TNF- $\alpha$ and IL-1 $\beta$ decreased the development of sepsis and also improved the survival rate in both animal models as well as in patients $[5,38]$. In the present work, a miR-133a-3p antagomir decreased the levels of proinflammatory cytokines in macrophages and sepsis-induced lung tissues, indicating that miR-133a-3p antagomir may exert a protective effect on lungs via modulating the expression of proinflammatory mediators. It was also evidenced that the treatment of miR-133a-3p antagomir suppressed the activity of MPO in lung tissues, which is an important biomarker for activation of neutrophils [39]. These findings suggest that miR-133a-3p antagomir may modulate infiltration of inflammatory cells. It may be possible that miR-133a$3 p$ can target polymorphonuclear neutrophils so as to decrease the MPO activity, which needs to be studied further. In the present study, the decreased infiltration of Evan's blue dye in the sepsis-induced lungs demonstrated decreased permeability of microvasculature of endothelium and also attenuated its integrity. These findings suggest that downregulated miR-133a-3p shows a proinflammatory effect in sepsis-mediated ARDS. There is increasing evidence suggesting an increase in epithelial or endothelial cell apoptosis in the development of ARDS [32, 40]. It has already been established that ARDS is accompanied by increased cell death, but the apoptotic inhibitors elevate the cell survival significantly in the sepsis model of ARDS [41, 42]. $\mathrm{Bcl}-2$, caspase-3 and Bax have been identified to play important roles in apoptotic cell death [43]; the findings were in agreement with this study and suggested a significant decrease in levels of 
A

mmu-miR-133a-3p

SIRT1 3'UTR
3' UGUCGACCAACUUCCCCUGGUU 5' \|\|\|\|
B

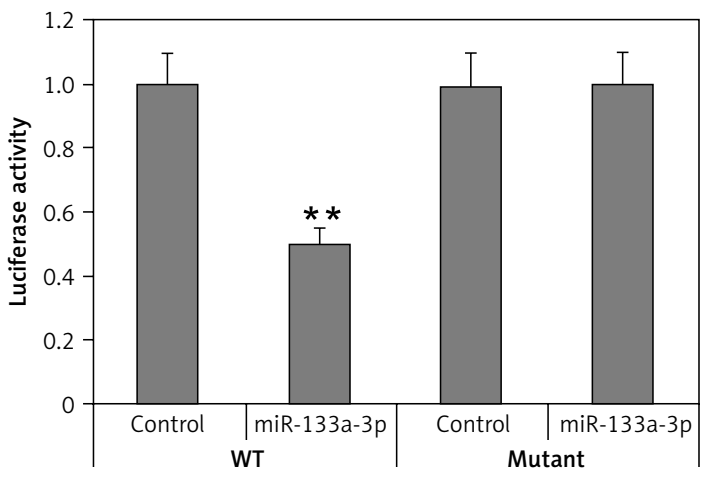

C


D

NC

miR-133a-3p antagomiR

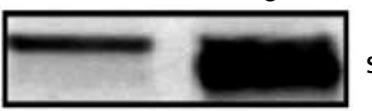

SIRT1
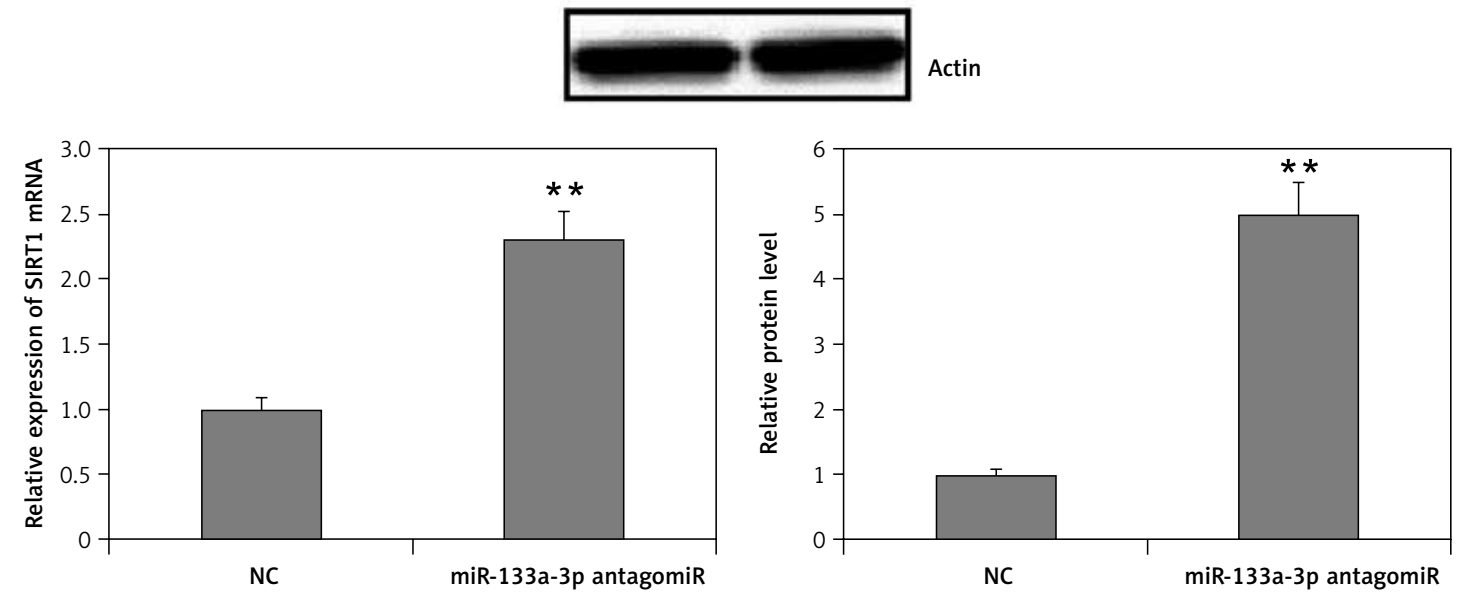

Figure 4. SIRT1 is a potential target for miR-133a-3p. A - The 3'UTR region of SIRT1 having a binding sequence with miR-133a-3p. Bioinformatics analysis identifies the interaction of SIRT1 with miR-133a-3p. B - The cells were cultured and received transfection with wild-type (WT) or mutated (MUT) SIRT1 3'UTR constructs of luciferase reporter followed by miR-133a-3p mimics for $48 \mathrm{~h}$ and then submitted to luciferase analysis. C - Immunoblotting and qRT-PCR analysis for expression of SIRT1 in alveolar macrophages which were transfected with negative control and agomir-133a-3p. D - qRT-PCR and Western blot evaluation of SIRT1 levels in alveolar macrophages transfected with negative control and miR-133a-3p antagomir

Data are presented as means $\pm S E(n=6) .{ }^{* *} P<0.01$ compared to negative control. 
A

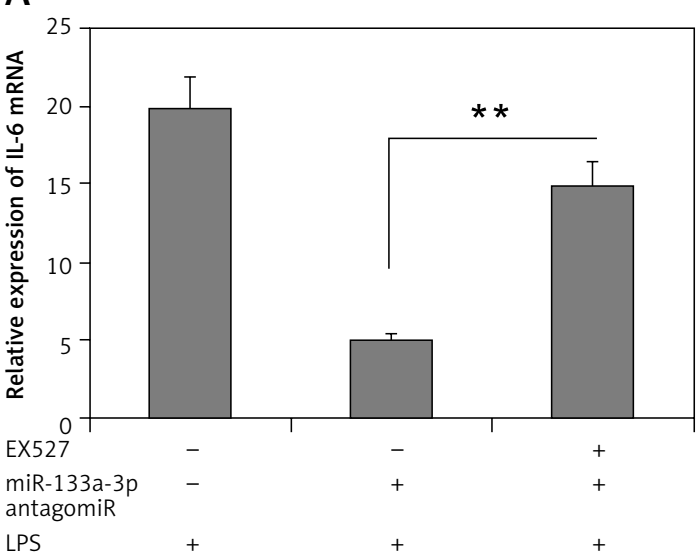

C

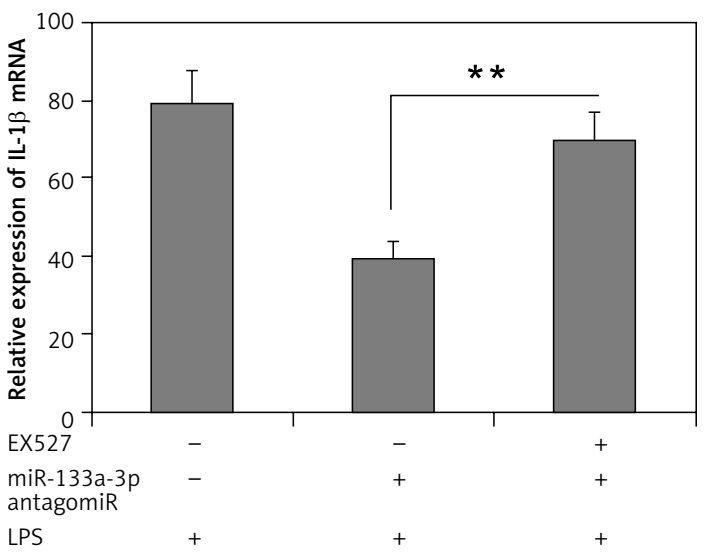

caspase- 3 and Bax in the lung tissues after treatment with miR-133a-3p antagomir. These results confirmed the apoptosis inhibitory activity of miR-133a-3p antagomir. Overall, these findings confirmed that miR-133a-3p antagomir exerts an attenuating effect in ARDS and can be an important therapeutic target for treating ARDS.

Sirtuin- 1 is also called NAD-dependent deacetylase sirtuin-1; it is a protein that in humans is converted by the SIRT1 gene. It is associated with cell growth, cell differentiation, metabolism and decrease of oxidative burden $[44,45]$. SIRT1 is reported to be responsible for anti-oxidative and anti-inflammatory effects, modulating apoptosis and inhibiting DNA damage in different types of cells. Reports have suggested a role of SIRT1 in relation to macrophages $[46,47]$. In a study recently, SIRT1 was found to play an attenuating role in burn-induced ARDS [48]. In the present study, it was demonstrated that SIRT1 is one of the favorable targets of miR-133a-3p; this finding was in agreement with a study in which SIRT1 was regulated by miR133a-3p and was confirmed to have potential binding sites for it [25]. In addition, pre-exposure of LPS-induced alveolar macrophages to SIRT1 inhibitor resulted in reversal of the anti-in-
B

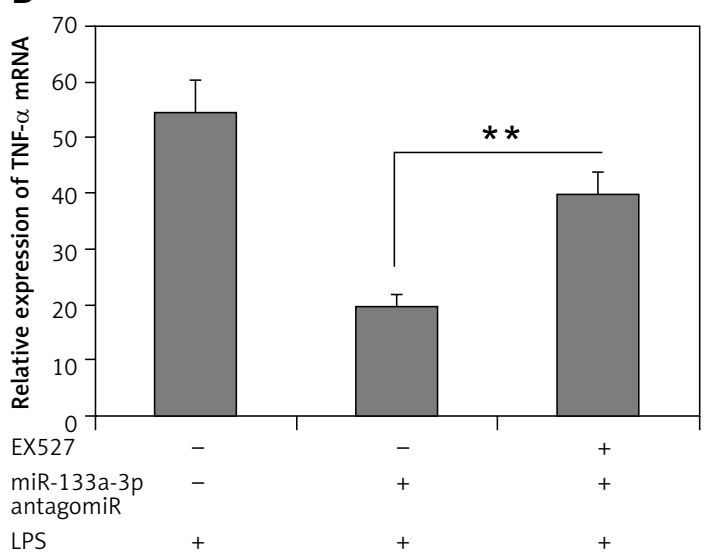

Figure 5. The SIRT1 inhibitor EX-527 inhibits the protective role of miR-133a-3p antagomir. A-C Results of qRT-PCR analysis demonstrating the mRNA levels of IL-1 $\beta$, IL- 6 and TNF- $\alpha$ in alveolar macrophages in presence and in absence of EX-527 after LPS + miR-133a-3p antagomir treatment

Data are presented as means $\pm S E(n=6)$. ${ }^{* *} P<0.01$ compared to group devoid of EX-527 treatment.

flammatory effect of miR133a-3p antagomir and also reversed the protective effect of miR-133a$3 p$ antagomir in a sepsis-mediated ARDS model. The findings hence suggested that miR-133a-3p regulates the sepsis-mediated ARDS via degradation of SIRT1. Altogether, the outcomes of bioinformatics and luciferase assay confirm that SIRT1 is a potential target gene of miR-133a-3p in sepsis-mediated ARDS.

The outcomes of the present work may be helpful for designing therapy for sepsis-induced ARDS. As it was evidenced that SIRT1 was a potential target, it would facilitate more efficient treatment of ARDS.

In conclusion, it was demonstrated for the first time that miR-133a-3p induces the expression of proinflammatory factors in a sepsis model of ARDS, and the protective role of miR-133a-3p antagomir in ARDS is due to its attenuating effect on secretion of inflammatory cytokines and inhibition of SIRT1-mediated apoptosis. These findings will definitely improve the knowledge about the mechanism involved in the regulatory effect of miR-133a-3p in sepsis-induced acute lung injury and will also guide us in developing new therapeutic agents for targeting miR-133a-3p. 


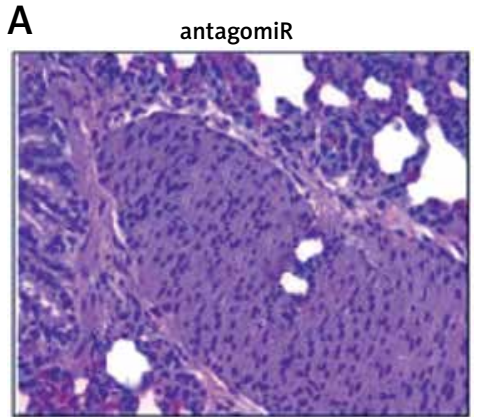

B

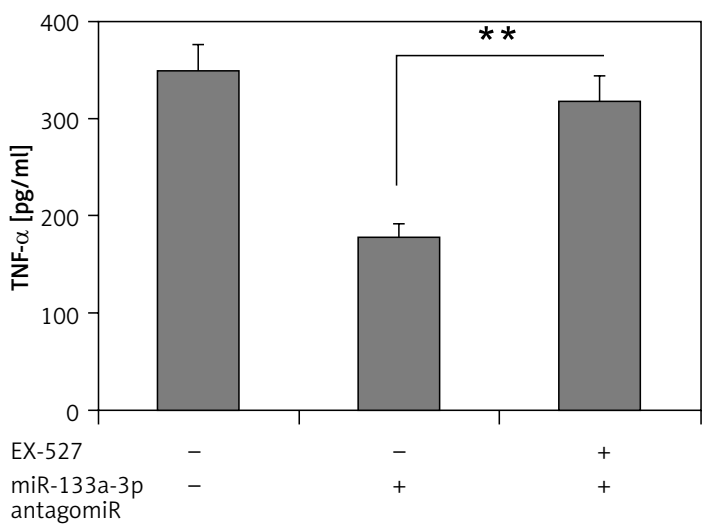

miR-133a-3p antagomiR

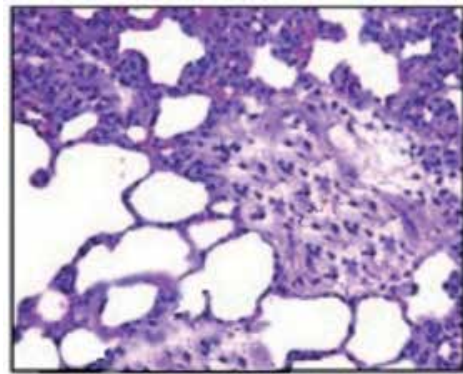

miR-133a-3p antagomiR + EX-527

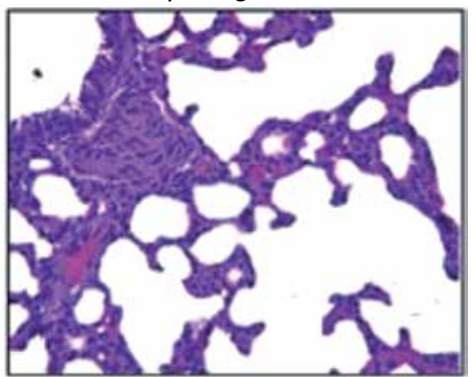

C

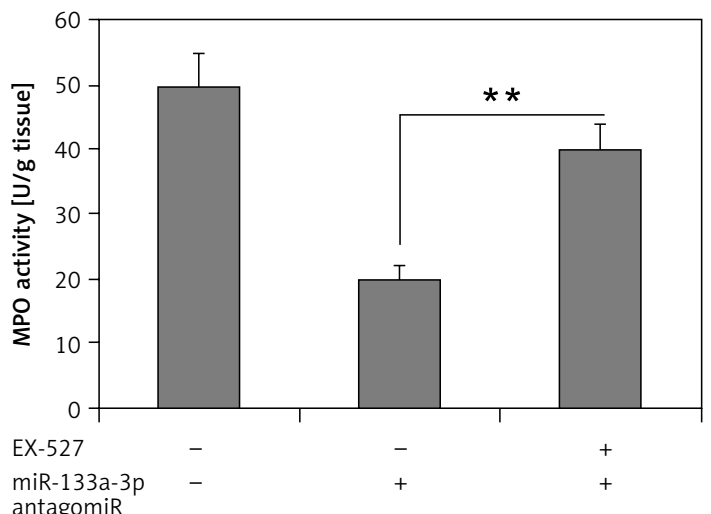

D

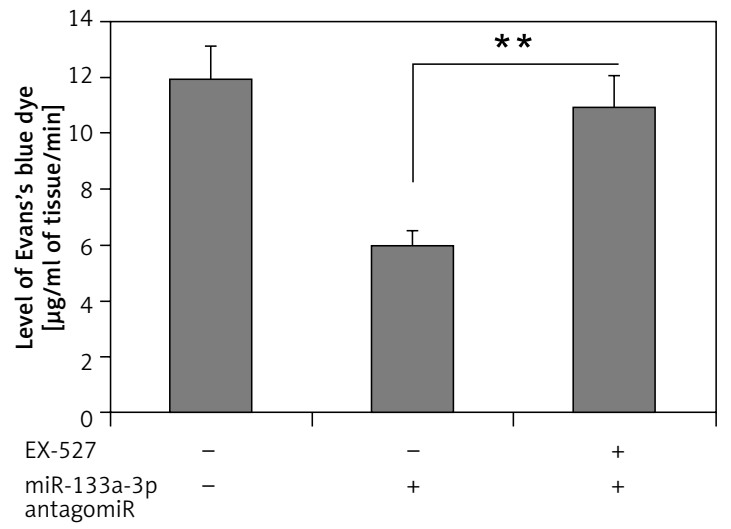

Figure 6. Effects of SIRT1 inhibitor EX-527 on the protective effect of miR-133a-3p antagomir in sepsis-induced lung injury. A - H\&E demonstrating the changes in morphology of the lung tissue induced by sepsis injury after $24 \mathrm{~h}$ of injury. The negative control miR-133a-3p antagomir sepsis injury group (NC-antagomir) demonstrated increase in lung edema, hemorrhage of alveoli, infiltration of neutrophils and damaged epithelial/endothelial cell structure compared to the sham operated group. The miR-133a-3p antagomir sepsis injury group (miR-133a-3p antagomir) demonstrated substantial attenuation on morphology of lung tissues, whereas the SIRT1 inhibitor EX527 (miR-133a-3p antagomir + EX-527) substantially inverted this improvement. (Scale - $50 \mu \mathrm{m}$ ) B - ELISA study demonstrated the effects of EX-527 treatment on the expression of TNF- $\alpha$ in sepsis-induced lung injury. $\mathbf{C}-$ Activities of MPO in sepsis injured lung tissues after $24 \mathrm{~h}$ of treatment of miR-133a-3p antagomir in presence or absence of without EX-527 in every group. D - Levels of Evan's blue dye were studied in each of the three; the results suggested that EX-527 reversed the decrease in levels of Evan's blue dye mediated by miR-133a-3p antagomir

The results are means $\pm S E(n=6)$. ${ }^{* *} P<0.01$. 


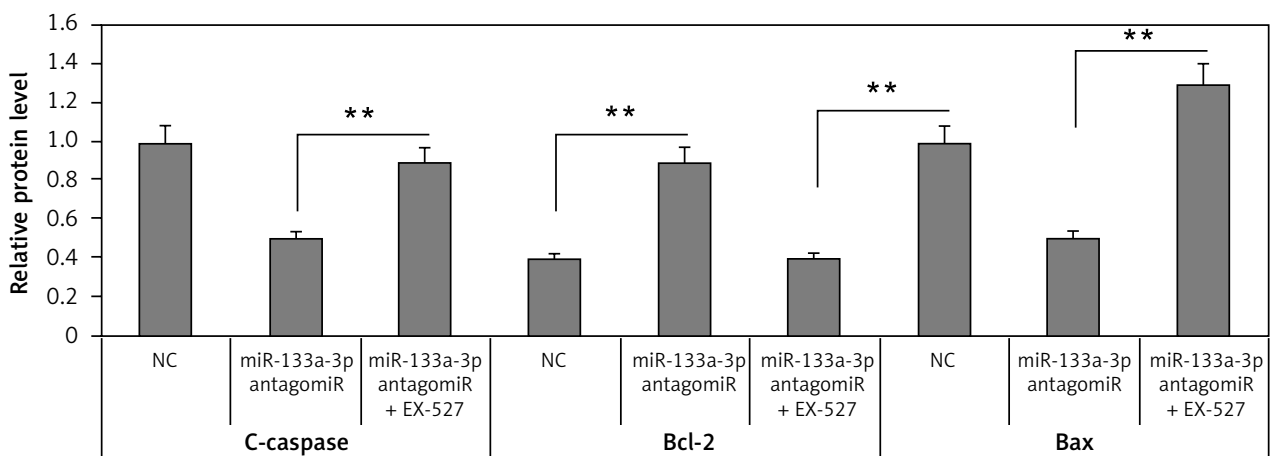

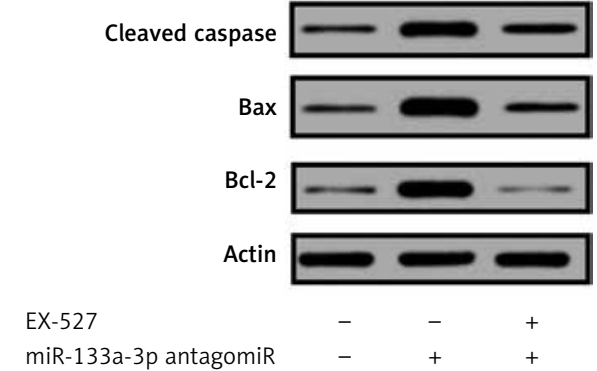

\section{Conflict of interest}

The authors declare no conflict of interest.

\section{References}

1. Fan $\mathrm{H}$, Zhao Y, Sun M, Zhu J. Urinary neutrophil gelatinase-associated lipocalin, kidney injury molecule-1, $\mathrm{N}$-acetyl-beta-D-glucosaminidase levels and mortality risk in septic patients with acute kidney injury. Arch Med Sci 2018; 14: 1381-6.

2. Abraham E, Singer M. Mechanisms of sepsis-induced organ dysfunction. Crit Care Med 2007; 35: 2408-16.

3. Avecillas JF, Freire AX, Arroliga AC. Clinical epidemiology of acute lung injury and acute respiratory distress syndrome: incidence, diagnosis, and outcomes. Clin Chest Med 2006; 27: 549-57.

4. Duran-Bedolla J, Montes de Oca-Sandoval MA, Saldaña-Navor V, Villalobos-Silva JA, Rodriguez MC, RivasArancibia S. Sepsis, mitochondrial failure and multiple organ dysfunction. Clin Invest Med 2014; 37: E58-69.

5. Rubenfeld GD, Caldwell E, Peabody E, et al. Incidence and outcomes of acute lung injury. N Engl J Med 2005; 353: 1685-93.

6. Sevransky JE, Levy MM, Marini JJ. Mechanical ventilation in sepsis induced acute lung injury/acute respiratory distress syndrome: an evidence-based review. Crit Care Med 2004; 32 Suppl: S548-53.

7. Wheeler AP, Bernard GR. Acute lung injury and the acute respiratory distress syndrome: a clinical review. Lancet 2007; 369: 1553-64.

8. Schultz MJ, van der Poll T. Animal and human models for sepsis. Ann Med 2002; 34: 573-81.

9. Kissoon N, Daniels R, van der Poll T, Finfer S, Reinhart K. Sepsis-the final common pathway to death from multiple organ failure in infection. Crit Care Med 2016; 44: e446.

10. Kim YS, Hwang JW, Jang JH, et al. Trapa japonica pericarp extract reduces LPS-induced inflammation in macrophages and acute lung injury in mice. Molecules 2016; 21: 392.
Figure 6. Cont. E - Western blot analysis for demonstrating the effect of EX-527 on protein levels of cleaved caspase-3, Bax, and Bcl-2 in sepsis injured lung tissues after $24 \mathrm{~h}$ of treatment

The results are means $\pm S E(n=6)$. ${ }^{* *} P<0.01$

11. Zhang J, Lee SM, Shannon S, et al. The type III histone deacetylase Sirt1 is essential for maintenance of T cell tolerance in mice. J Clin Invest 2009; 119: 3048-58.

12. Blander G, Guarente L. The Sir2 family of protein deacetylases. Annu Rev Biochem 2004; 73: 417-35.

13. Lavu S, Boss O, Elliott PJ, Lambert PD. Sirtuins: novel therapeutic targets to treat age-associated diseases. Nat Rev Drug Discov 2008; 7: 841-53.

14. Yang SR, Wright J, Bauter M, Seweryniak K, Kode A, Rahman I. Sirtuin regulates cigarette smoke-induced proinflammatory mediator release via RelA/p65 NF-kappaB in macrophages in vitro and in rat lungs in vivo: implications for chronic inflammation and aging. Am J Physiol Lung Cell Mol Physiol 2007; 292: L567-76.

15. Vidigal JA, Ventura A, The biological functions of miRNAs: lessons from in vivo studies. Trends Cell Biol 2015; 25: 137-47.

16. Oglesby IK, McElvaney NG, Greene CM. MicroRNAs in inflammatory lung disease - master regulators or target practice? Respir Res 2010; 11: 148.

17. Murdaca G, Tonacci A, Negrini S, et al. Effects of antagomiRs on different lung diseases in human, cellular, and animal models. Int J Mol Sci 2019; 20: pii: E3938.

18. Lagos-Quintana M, Rauhut R, Yalcin A, Meyer J, Lendeckel W, Tuschl T. Identification of tissue-specific microRNAs from mouse. Curr Biol 2002; 12: 735-9.

19. Koutsoulidou A, Mastroyiannopoulos NP, Furling D, Uney JB, Phylactou LA. Expression of miR-1, miR-133a, miR-133b and miR-206 increases during development of human skeletal muscle. BMC Dev Biol 2011; 11: 34.

20. Babiarz JE, Ravon M, Sridhar S, et al. Determination of the human cardiomyocyte mRNA and miRNA differentiation network by fine-scale profiling. Stem Cells Dev 2012; 21: 1956-65.

21. Wang LK, Hsiao TH, Hong TM, et al. MicroRNA-133a suppresses multiple oncogenic membrane receptors and cell invasion in non-small cell lung carcinoma. PLoS One 2014; 9: e96765.

22. Zhang W, Liu K, Liu S, Ji B, Wang Y, Liu Y. MicroRNA-133a functions as a tumor suppressor by targeting IGF-1R in hepatocellular carcinoma. Tumour Biol 2015; 36: 9779-88. 
23. Yoshino $\mathrm{H}$, Chiyomaru T, Enokida $\mathrm{H}$, et al. The tumour-sup pressive function of miR-1 and miR-133a targeting TAGLN2 in bladder cancer. Br J Cancer 2011; 104: 808-18.

24. Lan C, Shi X, Guo N, Pei H, Zhang H. Value of serum miR-155-5p and miR-133a-3p expression for the diagnosis and prognosis evaluation of sepsis. Zhonghua Wei Zhong Bing Ji Jiu Yi Xue 2016; 28: 694-8.

25. Shin AN, Han L, Dasgupta C, Huang L, Yang S, Zhang L. SIRT1 increases cardiomyocyte binucleation in the heart development. Oncotarget 2018; 9: 7996-8010.

26. Pinsky MR. Sepsis and multiple organ failure. Contrib Nephrol 2007; 156: 47-63.

27. Rossaint J, Zarbock A. Pathogenesis of multiple organ failure in sepsis. Crit Rev Immunol 2015; 35: 277-91.

28. Paunel-Görgülü A, Flohé S, Scholz $M$, Windolf J, Lögters T. Increased serum soluble Fas after major trauma is associated with delayed neutrophils apoptosis and development of sepsis. Crit Care 2011; 15: R20.

29. Laskin DL, Pendino KJ. Macrophages and inflammatory mediators in tissue injury. Annu Rev Pharmacol Toxicol 1995; 35: 655-77.

30. Kim YS, Hwang JW, Jang JH, et al. Trapa japonica pericarp extract reduces LPS-induced inflammation in macrophages and acute lung injury in mice. Molecules 2016; 21: 392.

31. Kissoon N, Daniels R, van der Poll T, Finfer S, Reinhart K. Sepsis-the final common pathway to death from multiple organ failure in infection. Crit Care Med 2016; 44: e446.

32. Hotchkiss RS, Karl IE. The pathophysiology and treatment of sepsis. N Engl J Med 2003; 348: 138-50.

33. Dolinay T, Kim YS, Howrylak J, et al. Inflammasome-regulated cytokines are critical mediators of acute lung injury. Am J Respir Crit Care Med 2012; 185: 1225-34.

34. Lolis E, Bucala R. Therapeutic approaches to innate immunity: severe sepsis and septic shock. Nat Rev Drug Discov 2003; 2: 635-45.

35. Parsons PE, Matthay MA, Ware LB, Eisner MD; National Heart, Lung, Blood Institute Acute Respiratory Distress Syndrome Clinical Trials Network. Elevated plasma levels of soluble TNF receptors are associated with morbidity and mortality in patients with acute lung injury. Am J Physiol Lung Cell Mol Physiol 2005; 288: L426-31.

36. Wang W, Liu Z, Su J, et al. Macrophage micro-RNA-155 promotes lipopolysaccharide-induced acute lung injury in mice and rats. Am J Physiol Lung Cell Mol Physiol 2016; 311: L494-506.

37. Law IKM, Bakirtzi K, Polytarchou C, et al. Neurotensin-regulated miR-133 alpha is involved in proinflammatory signalling in human colonic epithelial cells and in experimental colitis. Gut 2015; 64: 1095-104.

38. Vanden Berghe T, Demon D, Bogaert P, et al. Simultaneous targeting of IL-1 and IL-18 is required for protection against inflammatory and septic shock. Am J Respir Crit Care Med 2014; 189: 282-91.

39. Fialkow L, Fochesatto Filho L, Bozzetti MC, et al. Neutrophil apoptosis: a marker of disease severity in sepsis and sepsis-induced acute respiratory distress syndrome. Crit Care 2006; 10: R155.

40. Galani V, Tatsaki E, Bai M, et al. The role of apoptosis in the pathophysiology of acute respiratory distress syndrome (ARDS): an up-to-date cell-specific review. Pathol Res Pract 2010; 206: 145-50.

41. Kawasaki M, Kuwano K, Hagimoto N, et al. Protection from lethal apoptosis in lipopolysaccharide-induced acute lung injury in mice by a caspase inhibitor. Am J Pathol 2000; 157: 597-603.
42. Taneja R, Parodo J, Jia SH, Kapus A, Rotstein OD, Marshall JC. Delayed neutrophil apoptosis in sepsis is associated with maintenance of mitochondrial transmembrane potential and reduced caspase- 9 activity. Crit Care Med 2004; 32: 1460-9.

43. Morales-Cano D, Calviño E, Rubio V, et al. Apoptosis induced by paclitaxel via $\mathrm{Bcl}-2$, Bax and caspases 3 and 9 activation in NB4 human leukaemia cells is not modulated by ERK inhibition. Exp Toxicol Pathol 2013; 65: 1101-8.

44. Wang YQ, Cao Q, Wang F, et al. SIRT1 protects against oxidative stress-induced endothelial progenitor cells apoptosis by inhibiting $\mathrm{FOXO} 3$ a via FOXO3a ubiquitination and degradation. J Cell Physiol 2015; 230: 2098107.

45. Winnik S, Stein S, Matter CM. SIRT1 - an anti-inflammatory pathway at the crossroads between metabolic disease and atherosclerosis. Curr Vasc Pharmacol 2012; 10: 693-6.

46. Cohen HY, Miller C, Bitterman KJ, et al. Calorie restriction promotes mammalian cell survival by inducing the SIRT1 deacetylase. Science 2004; 305: 390-2.

47. Finkel T, Deng CX, Mostoslavsky R. Recent progress in the biology and physiology of sirtuins. Nature 2009; 460: 587-91.

48. Bai X, Fan L, He T, et al. SIRT1 protects rat lung tissue against severe burn-induced remote ALI by attenuating the apoptosis of PMVECs via p38 MAPK signaling. Sci Rep 2015; 5: 10277. 